\title{
Three-dimensional Analysis of the Attachment and Path of the Transverse Carpal Ligament
}

\author{
Mitsuhiko Nanno ${ }^{1}$, Takuya Sawaizumi ${ }^{2}$, Norie Kodera², \\ Yuji Tomori ${ }^{2}$ and Shinro Takai ${ }^{2}$ \\ ${ }^{1}$ Department of Orthopaedic Surgery, Nippon Medical School Musashi Kosugi Hospital \\ ${ }^{2}$ Department of Orthopaedic Surgery, Nippon Medical School
}

\begin{abstract}
Purpose: The purpose of this study was to describe and evaluate the detailed anatomic locations and areas of ligamentous attachments and paths of the transverse carpal ligament (TCL) on a threedimensional (3-D) surface model.
\end{abstract}

Methods: Ten fresh-frozen cadaver wrists were used to dissect and identify the TCL. Their ligament attachments and whole bone surfaces were digitized three-dimensionally and their areas evaluated. The attachments of each ligament were represented in a model combining CT surfaces overlaid by a digitized 3-D surface, and were also visually depicted with a different color for each on 3-D images of the bones.

Results: The TCL was found to be composed of two or three discrete ligaments. Both the trapeziumhook of hamate ligament and the trapezium-pisiform ligament were identified in all ten specimens. The scaphoid-pisiform ligament was found in only two of the ten specimens. The average areas of the attachments of the TCL were $42.7 \mathrm{~mm}^{2}$ on the trapezium, $30.0 \mathrm{~mm}^{2}$ on the hook of hamate, $21.6 \mathrm{~mm}^{2}$ on the pisiform, and $12.7 \mathrm{~mm}^{2}$ on the scaphoid.

Conclusions: The anatomic 3-D attachment sites of the TCL were visually shown qualitatively, and their areas quantified. This 3-D information offers further knowledge and understanding of the anatomy and biomechanics of the TCL. It could also help in the accurate assessment of radiographic images and treatment of various wrist injuries and diseases when performing such procedures as carpal tunnel release, Guyon's canal release, trapeziectomy, hook of hamate excision, or arthroscopy.

(J Nippon Med Sch 2015; 82: 130-135)

Key words: anatomy, attachment, transeverse carpal ligament, carpal tunnel, hand

\section{Introduction}

The transverse carpal ligament (TCL) serves the palmar roof of the carpal tunnel by attaching laterally to the ridge of the trapezium and the scaphoid tuberosity, and medially to the hamate and pisiform ${ }^{1}$. To decompress the carpal tunnel, transecting the TCL is the standard surgical treatment for patients with carpal tunnel syndrome ${ }^{2}$. However, the specific anatomic description of the TCL remains incompletely understood, and the TCL is not typically visualized completely. Recently, Manley et al. ${ }^{3}$ used digital photography and computer-aided image analysis to describe and quantify the two-dimensional footprint of the TCL attachment sites on the carpal bones. However, there has been little information detailing the specific location and area of ligamentous attachments on the threedimensional (3-D) surface of the metacarpals and carpal bones $^{4,5}$. Currently, a number of techniques for carpal tunnel release have been reported. Moreover, there is a trend toward less invasive surgery and smaller incisions. In addition, poor visualization and the risk for complication, including incomplete ligament release, inability to identify anatomic variations, and iatrogenic nerve injury while performing the endoscopic carpal tunnel release ${ }^{6}$ have also been described. Visually demonstrating the at-

Correspondence to Mitsuhiko Nanno, Department of Orthopaedic Surgery, Nippon Medical School Musashi Kosugi Hospital, 1396 Kosugi-cho, Nakahara-ku, Kawasaki, Kanagawa 211-8533, Japan

E-mail: nanno-mi@ga2.so-net.ne.jp

Journal Website (http://www.nms.ac.jp/jnms/) 
tachment sites of the ligaments on 3-D images of the bone can help to provide a better understanding of the anatomy and orientation of ligaments.

The purpose of this study was to detail the precise anatomical description of the attachments and paths of the TCL using a combination of meticulous dissection, computed tomography (CT), and a 3-D digitization technique. This 3-D information could improve the knowledge and understanding of the normal anatomy and its impact on the mechanics of the TCL, and help in the assessment and treatment of various wrist injuries and diseases.

\section{Materials and Methods}

Ten fresh-frozen cadaver upper extremities (eight males and two females, five pairs, aged 53-77 years, average age $=64.6$ years) were used in this study. The transverse carpal ligament (TCL) of all ten specimens was carefully dissected by volar approaches using loupe magnification. The carpal tunnel and the Guyon's canal were dissected. All components of the TCL were identified and categorized, and the length, width, and thickness of each component were measured using a precision caliper accurate to $0.1 \mathrm{~mm}$. After measurement, the flexor tendons and the median nerve in the carpal tunnel were excised to distinguish the accurate sites of ligamentous attachments. Each component of the TCL was carefully removed from the carpal bones, and its attachments were marked with an oil-based color marker. Three $1.5 \mathrm{~mm}$ screws were placed into the individual metacarpal and carpal bones as markers to define the 3-D local coordinate system. In addition, one to two single half pins were inserted into each bone to ensure it was firmly fixed to the digitizing platform.

Both the making of the 3-D digitized surface models with the ligament attachments and the showing of the ligament attachments on 3-D CT models of the bones were performed according to methods already reported ${ }^{4,5}$. A MicroScribe-3DX Digitizer (Immersion Corp., San Jose, CA) and 3-D surface reconstruction software (Spider; developed by Orthopaedic Biomechanics laboratory, The University of Texas Medical Branch, Galveston, TX) were used to manually digitize the ligament attachments and whole bone surfaces at approximately $0.67 \mathrm{~mm}$ intervals and to compute their areas. The accuracy of this digitizer is $0.23 \mathrm{~mm}$. Each ligament attachment was marked with a different color. The resulting sets of digitized points were modeled as triangulated surfaces automatically with the Spider software. Surface areas for each ligament attachment were defined by summing the corresponding triangles. The ligament attachment areas were evaluated in square millimeters $\left[\mathrm{mm}^{2}\right]$ (mean $\left.\pm \mathrm{SD}\right)$. The triangulated surface of these digitized 3-D models was displayed using a combination of Spider and Magics $X$ (Materialise Corp., Ann Arbor, MI) software programs.

CT images of the wrists were taken at $0.67 \mathrm{~mm}$ intervals by a GE 9800 CT scanner (General Electric, Milwaukee, MI) before dissection in selected specimens. 3-D CT models of each bone were generated from the CT images using software (Mimics 9.0 and Magics X; Materialise, Ann Arbor, MI). These digitized models, with their corresponding digitized surfaces and their associated regions of ligament attachment, were overlaid on the 3-D CT models preserving the $\mathrm{CT}$ coordinate space to clarify the relationship of ligament attachments between different bones using the Magics software. The attachments of each ligament were visually shown with an individual identifying solid color on the 3-D images of the bones, while the paths of the ligaments were described in transparent colors.

\section{Results}

Careful dissection revealed that the TCL attached to the ridge of the trapezium and the scaphoid tuberosity on the radial side, and to the hook of the hamate and the pisiform on the ulnar side (Fig. 1). According to the running directions of the ligament and the ligament attachments on the carpal bone, three components of the TCL were identified: the trapezium-hook of hamate ligament (THL), the trapezium-pisiform ligament (TPL), and the scaphoid-pisiform ligament (SPL). Both the THL and the TPL were identified in all ten specimens. On the other hand, the SPL was found in only two of the ten specimens (Fig. 2). The THL, TPL, and SPL were located from superficial to deep, respectively, on the volar side of the wrist (Fig. 3A, B). The THL originated from the top of the tubercle of the trapezium and attached to the edge of the hook of the hamate. The TPL originated from the top to the ridge of the trapezium and attached to the radial edge of the pisiform. The SPL originated from the top to the scaphoid tuberosity and attached to the radial edge of the pisiform. The Guyon's canal, which contains the ulnar nerve and artery, runs superficial to the TCL.

The results of manual measurements of each ligament's length, width, and thickness were described in millimeters $[\mathrm{mm}]($ mean $\pm \mathrm{SD})$ (Table 1). The average length of the THL, TPL, and SPL were $21.8 \pm 3.7 \mathrm{~mm}, 27.2 \pm 5.4 \mathrm{~mm}$, and $24.3 \pm 6.4 \mathrm{~mm}$ respectively. The average width of the 


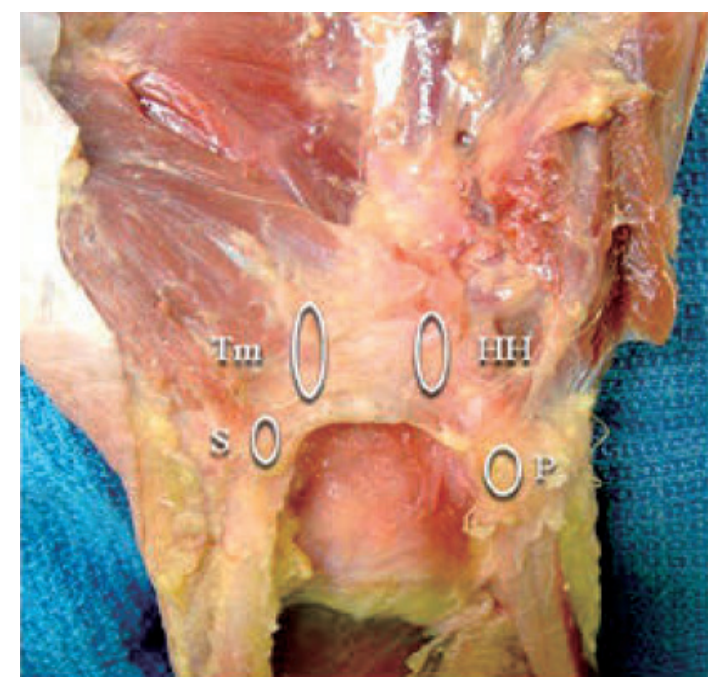

Fig. 1 Identification of the transverse carpal ligament and distinguishing of the ligament attachments Tm: Trapezium, HH: hook of hamate, P: pisiform, S: scaphoid

THL, TPL, and SPL were $13.4 \pm 2.1 \mathrm{~mm}, 6.53 \pm 0.93 \mathrm{~mm}$, and $5.33 \pm 2.3 \mathrm{~mm}$ respectively. The average thickness of the THL, TPL, and SPL were $2.18 \pm 0.22 \mathrm{~mm}, 1.27 \pm 0.16$ $\mathrm{mm}$, and $1.14 \pm 0.31 \mathrm{~mm}$ respectively. The average width and thickness of the THL were larger than those of the other two components of the TCL. On the other hand, the average length of the TPL was the largest of three components of the TCL.

The average attachment areas of the TCL were $42.7 \pm$ $18.8 \mathrm{~mm}^{2}$ on the trapezium, $30.0 \pm 11.7 \mathrm{~mm}^{2}$ on the hook of the hamate, $21.6 \pm 8.37 \mathrm{~mm}^{2}$ on the pisiform, and $12.7 \pm$ $2.83 \mathrm{~mm}^{2}$ on the scaphoid (Fig. 4). The average sizes of the whole surface area of trapezium, hamate, pisiform, and scaphoid were $1,215.5 \pm 160.9 \mathrm{~mm}^{2}, 1,445.1 \pm 249.4 \mathrm{~mm}^{2}$, $538.4 \pm 53.2 \mathrm{~mm}^{2}$, and $25.0 \pm 208.6 \mathrm{~mm}^{2}$, respectively. The area of the TCL attachment on each bone was calculated as a percentage of the total surface area of that bone. The areas of the TCL attachment on the trapezium, the hook of the hamate, the pisiform, and the scaphoid respectively occupied $3.5 \%, 2.1 \%, 4.0 \%$, and $1.0 \%$ of the average whole surface area of each bone.

\section{Discussion}

The TCL covers the volar side of the carpal tunnel and spans from the trapezium and the scaphoid radially to the hook of the hamate and the pisiform ulnarly ${ }^{1}$. Currently, several studies on the anatomy of the TCL regarding the carpal tunnel have been published. However, detailed anatomic descriptions of the TCL and its associated anatomy are not yet complete ${ }^{7-13}$. Most previous studies

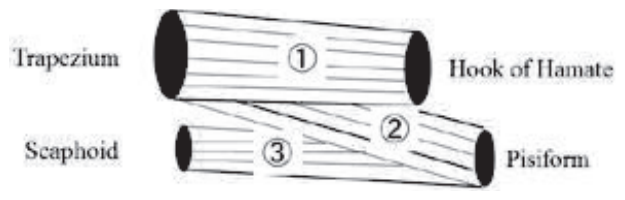

Fig. 2 Three components of the transverse carpal ligament

have quantitatively identified the TCL thickness, length, width, and volume, and its variations, and described the morphology of the carpal tunnel ${ }^{7-13}$. Tanzer ${ }^{7}$ first reported the thickness of the TCL. Subsequently, Johnson et al. ${ }^{8}$, Cobb et al. ${ }^{9}$, Tanabe et al. ${ }^{10}$, and Brett et al. ${ }^{11}$ reported and further detailed the dimensions of the TCL. Pacek et al. ${ }^{12}$ also described the average thicknesses and width of the TCL as $14.4 \mathrm{~mm}$ and $2.1 \mathrm{~mm}$, respectively. In the current study, similar results were obtained. In addition, despite a considerable increase in knowledge about the TCL, there have been several inconsistencies in the anatomic description of this ligament. Cobb et al. ${ }^{9}$ redefined the palmar boundary of the carpal tunnel to include three continuous segments of flexor retinaculum: the thin proximal segment composed of thickened deep investing fascia of the forearm; the TCL proper; and the distal segment of the flexor retinaculum, composed of an aponeurosis between the thenar and hypothenar muscles. What Cobb et al. ${ }^{9}$ described as the central portion of the flexor retinaculum has subsequently been termed the TCL. Isogai et al. ${ }^{14}$ classified the TCL into four types, according to the running directions and the location of the ligamentous attachments. Their Type I, in which distal transverse and ulnar oblique laminae were observed, was the most common $(44.2 \%)$. On the other hand, their type III $(10.4 \%)$, in which distal and proximal transverse, and ulnar oblique laminae were observed, was a minority. In the current study, the distal and proximal transverse, and ulnar oblique laminae as described by Isogai et al. were considered to correspond to the THL, TPL, and SPL, respectively. Moreover, Type I and Type III in Isogai's classification were identified in eight and two of our ten specimens, respectively.

In the present study, the THL was the thickest and widest ligament of the three components of the TCL, and was found in all specimens. Moreover, the area of the THL attachment on the individual carpal bone was the largest of the three components of the TCL. This suggests that the main segment of the TCL is the THL and that the THL plays an important role in the maintenance and dorso-palmar stability of the transverse carpal arch of the 

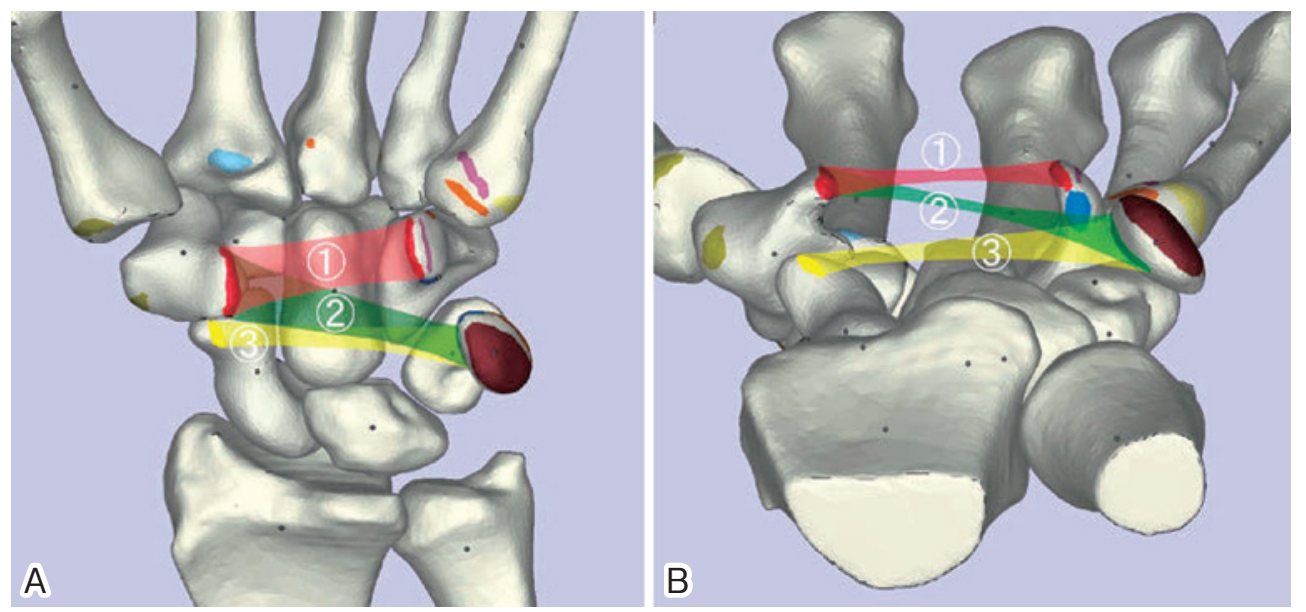

Fig. 3 A, B: A palmar view of a 3-D model of a left wrist.

The attachments and the paths of the transverse carpal ligament

(1) Trapezium-Hook of hamate segment

(2) Trapezium-Pisiform segment

(3) Scaphoid-pisiform segment

The solid colored areas show ligament attachment locations, and the transparent colored areas show the paths of the ligaments.

Table 1 Dimensions of three components of the transverse carpal ligament

\begin{tabular}{lcccc}
\hline \multicolumn{1}{c}{ Component of the ligament } & Length $(\mathrm{mm})$ & Width $(\mathrm{mm})$ & Thickness $(\mathrm{mm})$ & Number of cases \\
\hline (1) Trapezium-Hook of hamate ligament & $21.8 \pm 3.7$ & $13.4 \pm 2.1$ & $2.18 \pm 0.22$ & 10 \\
(2) Trapezium-Pisiform ligament & $27.2 \pm 5.4$ & $6.53 \pm 0.93$ & $1.27 \pm 0.16$ & 10 \\
(3) Scaphoid-Pisiform ligament & $24.3 \pm 6.4$ & $5.33 \pm 2.3$ & $1.14 \pm 0.31$ & 2 \\
\hline
\end{tabular}

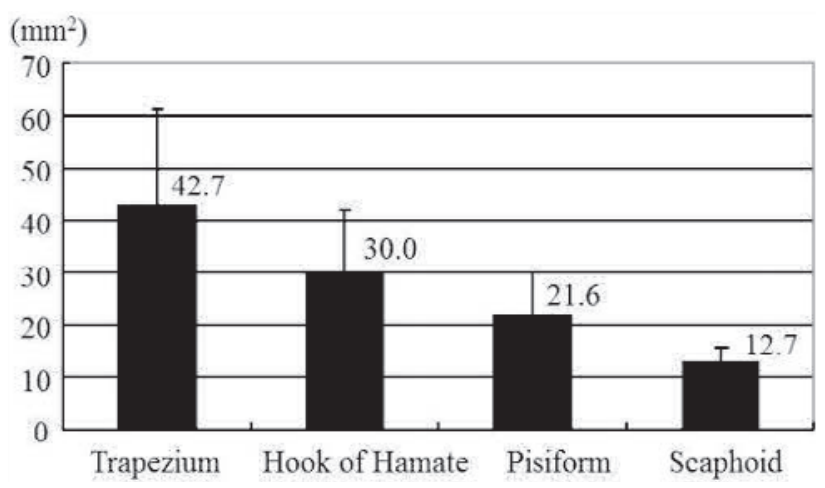

Fig. 4 The attachment area of the transverse carpal ligament on each carpal bone

hand. On the other hand, the SPL, which was found in only two of the ten specimens, was relatively thin and narrow. It is thought that the SPL may play only a minor role in the stability of the TCL.

We have previously described the average sizes of all ligamentous attachments except that of the TCL on each carpal bone $e^{4}$. In the current study, the attachment size of the TCL on the individual carpal bone was defined. Evaluating the ligamentous attachment area on the basis of our findings, we found that the attachment area of the TCL on each carpal bone was bigger than those of the other ligaments. Furthermore, the average size of the TCL attachment area on each bone in order of largest to smallest was trapezium $>$ hook of the hamate $>$ pisiform $>$ scaphoid. From this study it can be speculated that the TCL provides the main stability of the trapezium and the hook of the hamate.

Carpal tunnel syndrome is one of the most common entrapment neuropathies of the upper extremity. This occurs when the median nerve is compressed within the carpal canal ${ }^{16}$. Transection of the TCL to decompress the carpal tunnel has been the most standard surgery for carpal tunnel syndrome ${ }^{2}$. A number of carpal tunnel releases, including traditional open release, limited incision open release, and endoscopic release, have been described $^{2,6,15}$. Currently, the applications of more minimally invasive techniques for the carpal tunnel release have gradually increased, to avoid pillar pain, hypertrophic scarring and scar hypersensitivity ${ }^{2,6,15}$. However, these less invasive and smaller incisions may result in an incomplete release of the TCL due to poor visualization, lim- 


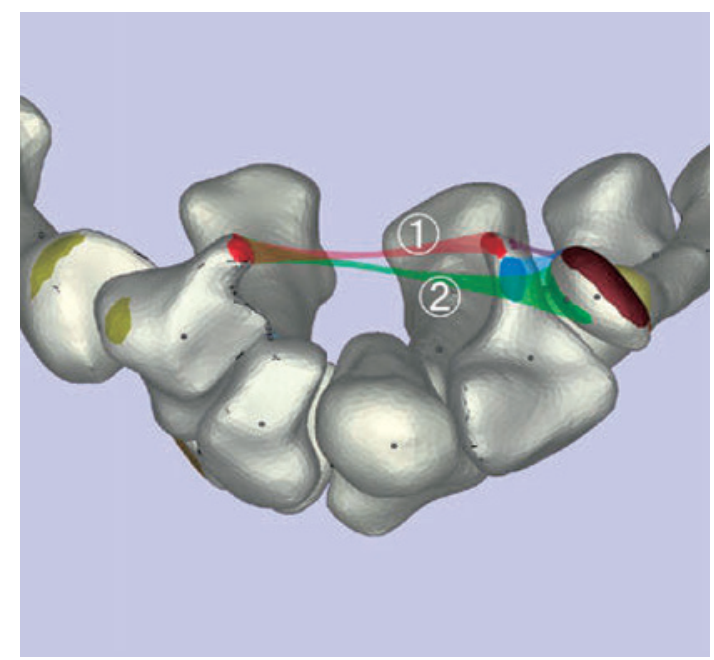

Fig. 5 A carpal tunnel view of a 3-D model of a left wrist. The attachments and the paths of the transverse carpal ligament in carpal tunnel view

(1) Trapezium-Hook of hamate portion, (2) Trapezium-Pisiform portion

The solid colored areas show ligament attachment locations, and the transparent colored areas show the paths of the ligaments.

ited exposure, and an inability to identify anatomic variations of the $\mathrm{TCL}^{2,6,15}$. Accurate knowledge of ligament attachment areas and landmarks is essential to prevent injury to adjacent structures with greater confidence and safety, while performing the carpal tunnel release $\mathrm{e}^{2,6,15}$.

Recently, Manley et al. ${ }^{3}$ quantitatively defined the twodimensional anatomic ligament attachment site of the TCL to the carpal bones using digital photography and computer-aided image analysis. They found that the average area of the ligament attachment site of the trapezium was $42 \mathrm{~mm}^{2}$, hamate $40 \mathrm{~mm}^{2}$, pisiform $38 \mathrm{~mm}^{2}$, and scaphoid $29 \mathrm{~mm}^{2}$. Naturally, the accurate anatomical location and area of ligamentous attachments on the individual bones have a 3-D surface morphology. Further clarification and details of location of the TCL are needed to understand the anatomy and function of this ligament, particularly the 3-D location of the ligament attachments. However, there has been little information that details the specific location and area of ligamentous attachments on the 3-D surface of the metacarpals and carpal bones ${ }^{4,5}$.

In the current study, the anatomic 3-D attachment sites and paths of three components of the TCL were visually demonstrated qualitatively and their areas quantified using a combination of meticulous dissection, CT imaging, and 3-D digitization. The location of the three components of the TCL is complicated. The THL, TPL, and SPL were situated on the volar side of the wrist superficial to deep, respectively. Additionally, the TCL was not only the roof of the carpal tunnel, but also the bottom of the Guyon's canal with both the piso-hamate ligament and the 5th metacarpal-hamate ligament (Fig. 5). The spatial orientation of these ligaments was obtained from the anatomical 3-D description of the attachment sites and the paths of the TCL. Especially in patients with the SPL, it may be difficult to identify the proximal portion of the TCL while performing carpal tunnel release with minimal incision. Generally, it is thought to be easy to identify the two bony landmarks (the trapezium and hook of hamate). In the current study, the THL attached to the two landmarks was situated most superficially. Release of this THL would ensure the easy and complete release of the TPL and the SPL, which are deeper than the THL. The 3-D information could assist in a better knowledge and understanding of the anatomy and biomechanics of the normal TCL. It could also help to improve diagnostic image analysis and treatment of various wrist injuries and diseases when performing carpal tunnel release, Guyon's canal release, trapeziectomy, hook of hamate excision, or arthroscopy.

There are some limitations to this study. First, the number of specimens used was small. A greater number of specimens would better clarify variations in the dimensions and the attachment areas of the TCL. Second, the boundary of the ligament attachment was manually defined and marked, and measurement of the dimensions of the elastic ligament may be difficult. However, all specimens were meticulously dissected using loupe magnification, and the dimensions of each ligament were measured by the same examiner three times. The average of the three measurements was calculated for each parameter. In addition, the ligament attachment areas on the bone were easily identified. Therefore, we think that the measured dimensions and areas were accurate.

Conflict of Interest: The authors declare no conflict of interest.

Acknowledgements: The authors gratefully acknowledge the help of Steven F. Viegas, MD and William L. Buford, Ph.D for their assistance with this study.

\section{References}

1. Stecco C, Macchi V, Lancerotto L, Tiengo C, Porzionato A, De Caro R: Comparison of transverse carpal ligament and flexor retinaculum terminology for the wrist. J Hand Sureg 2010; 35: 746-753.

2. Palmer DH, Paulson JC, Lane-Larsen CL, Peulen VK, Ol- 
son JD: Endoscopic carpal tunnel release: a comparison of two techniques with open release. Arthroscopy 1993; 9: 498-508.

3. Manley M, Boardman M, Goitz RJ: The carpal insertions of the transverse carpal ligament. J Hand Surg 2013; 38: 729-732.

4. Nanno M, Buford WL, Patterson RM, Andersen CR, Viegas SF: Three-dimensional analysis of the ligamentous attachments of the second through fifth carpometacarpal joints. Clin Anat 2007; 20: 530-544.

5. Nanno $M$, Buford WL, Patterson RM, Andersen CR, Viegas SF: Three-dimensional analysis of the ligamentous attachments of the first carpometacarpal joint. J Hand Surg 2006; 31: 1160-1170.

6. Levy HJ, Soifer TB, Kleibart FA, Lemak LJ, Bryk E: Endoscopic carpal tunnel release: An anatomical study. Arthroscopy 1993; 9: 1-4.

7. Tanzer RC: The carpal syndrome. J Bone Joint Surg 1959; 41: 626-634.

8. Johnson RK, Shrewsbury MM: Anatomical course of the thenar branch of the median nerve -- usually in a separate tunnel through the transverse carpal ligament. J Bone Joint Surg 1970; 52: 269-273.

9. Cobb TK, Dalley BK, Posterano RH, Lewis RC: Anatomy of the flexor retinaculum. J Hand Sureg 1993; 18: 91-99.

10. Tanabe T, Okutsu I: An anatomical study of the palmar ligamentous structures of the carpal canal. J Hand Surg 1997; 22: 754-757.

11. Brett AW, Oliver ML, Agur AM, Edwards AM, Gordon KD: Quantification of the transverse carpal ligament elastic properties by sex and region. Clin Biomech 2014; 29: 601-606.

12. Pacek CA, Chakan M, Goitz RJ, Kaufmann RA, Li ZM: Morphological analysis of the transverse carpal ligament. Hand 2010; 5: 135-140.

13. Rotman MB, Donovan JP: Pratical anatomy of the carpal tunnel. Hand Clinics 2002; 18: 219-230.

14. Isogai S, Murakami G, Wada T, Akita K, Yamashita T, Ishii S: Laminar configuration of the transeverse carpal ligament. J Orthop Sci 2002; 7: 79-83.

15. Gelberman RH, Hergenroeder PT, Hargens AR, Lundborg GN, Akeson WH: The carpal tunnel syndrome. A study of canal pressures. J Bone Joint Surg 1981; 63: 380-383.

(Received, October 28, 2014)

(Accepted, February 17, 2015) 